\title{
Dielectric flow sensing in resin transfer moulding of carbon fibre reinforced composites
}

\author{
Alexandros A Skordos ${ }^{1}$ and Ivana K Partridge ${ }^{2}$ \\ Advanced Materials Department, School of Industrial and Manufacturing Science \\ Cranfield University, Bedford, MK43 OAL,UK
}

\begin{abstract}
An in-situ dielectric technique for sensing flow is outlined, and shown to be suitable for use in moulding of composites from liquid resins containing continuous conducting fibres. This work builds upon the recent development of a sensor for monitoring flow and cure in thermosetting composites with (non-conductive) glass reinforcements. The linear relationship between the position of the flow front in the mould and the electrical admittance of the sensor system, which is observed in resins with non-conductive reinforcements, is found to extend to resins containing conducting fibres. The performance of the sensor in detecting the position of the flow front is evaluated by comparison with visual observations. The capability of the sensing system to monitor the cure of the resin after mould filling is also investigated. The results demonstrate that the sensor can both locate the flow front accurately and monitor the cure, identifying vitrification in a way that is similar to conventional microdielectrometry. Data from flow measurements are output in complex number format, which offers the potential to produce a (method for self-assessment) method than can evaluate qualitatively the performance of the sensor system.
\end{abstract}

Keywords: flow monitoring, cure monitoring, liquid moulding, dielectric sensors, impedance, carbon reinforcements

\section{Introduction}

Liquid moulding processes for the manufacture of continuously reinforced composites have received increased attention in recent years. In comparison with conventional processes, which

\footnotetext{
${ }^{1}$ Electronic address: : a.a.skordos@cranfield.ac.uk

${ }^{2}$ Electronic address: i.k.partridge@carnfield.ac.uk
} 
involve laying up and autoclaving, they offer a potentially cost-efficient and easily automated alternative. Various liquid moulding processes, using either rigid tooling (e.g. resin transfer moulding - RTM) or flexible tooling (e.g. vacuum infusion) have been developed. In all cases, the central characteristic of these processes is that the filling/consolidation stage is separated from the curing stage, and becomes the critical phase of the manufacturing process. Therefore, resin filling and the phenomena associated with it have become points of focus for research on composites manufacturing. Models attempting to simulate the impregnation of liquid resin into a dry fabric have been developed by several groups [1-4]. These models are all based on the application of Darcys' law, which assumes that the rate at which a fluid flows through a porous medium is proportional to the pressure gradient. The proportionality coefficient (permeability) has been the subject of extensive study, and numerous investigations have addressed issues related to its practical measurement [5-10]. It has been shown that the reproducibility and repeatability of permeability measurements on composite reinforcements are poor $[11,12]$. Consequently, the performance of flow models that use permeability as the fundamental parameter is similarly limited.

Methods for in-situ flow monitoring, which are adaptable to the industrial environment, were first investigated as an alternative to the modelling approach. These methods can be divided into two main categories; (i) discrete methods which record the instant at which the resin arrives at a specific location; and (ii) continuous methods which record the response of a monitoring system that detects the current position of the resin flow front .

In discrete methods, a sensing system that responds to the arrival of the resin is placed at specific locations in the mould. This technique was first implemented by Kranbuehl and co-workers [13]. Their system comprised a number of interdigitated dielectric sensors placed on the bottom face of an RTM mould. The arrival of the flow front caused a discrete jump in dielectric properties when the resin came into contact with the sensor. Similar systems, based on the abrupt change of DC conductivity that occurs when a liquid polymer shorts out the space between the ends of two conductive wires, have been developed by other workers[14]. The progress of in-plane filling in RTM has also been monitored using a grid of electric wires arranged in two sets, placed orthogonally at some distance from each other [15]. With appropriate multiplexing, it is then possible to determine whether a specific node of the grid has been covered by resin. In all cases, the discrete character of these measuring techniques limits their applicability since, in order to 
acquire a global view of the flow patterns in a complicated component, large numbers of point sensors are required. This was the reason for the investigation of continuous flow sensing techniques, which resulted in the development of several new methods. The measurement of changes in DC conductivity between two wires running along the mould has proved adequate for the location of flow fronts [16]. Similarly, a fibre optic technique based on the fluorescence of a dye dissolved in the resin has been used to determine the percentage of a fibre covered by the resin during filling [17]. This technique uses the evanescent field that is created at the interface between the fibre and the surrounding medium as a result of interference between the incoming and reflected rays. Recently, a new technique, based on the application of an AC voltage between two flat wires running along the mould, has been demonstrated by the present authors to be capable of locating the position of the flow front accurately. The sensing configuration also provided a means of monitoring cure in the resin during subsequent heating of the mould [18]. All of these techniques are applicable to the manufacture of composites with non-conductive reinforcements. However, the presence of carbon fibre, which is the reinforcement used in most advanced composites, would disturb both the electric field in dielectric sensing and the evanescent field in fibre optic sensing, and would cause shorting between the wires in DC conductivity sensing.

In this investigation, the recently published work on dielectric flow sensing [18] is extended to composites containing conductive reinforcements. A novel dielectric system is devised and evaluated experimentally, as both a flow detector and a macroscopic device for cure monitoring.

\section{Sensor setup}

\subsection{Principle of operation}

The proposed sensor system comprises an array of thin insulated wires, which are in contact with the carbon preform and are connected to an impedance analyzer. The reinforcement is also connected to the analyzer, and the measurement is performed between the array of wires and the fibres of the preform. A schematic diagram of the arrangement of fibre tows and wires in the novel sensor system is presented in Fig. 1. In the case illustrated, the wires are in contact with the tool. As the fibre tows are displaced by the hard wires, some gaps are formed. With the application of a voltage, an electric field is formed between the conductive core of each wire and the adjacent fibres. The field occupies the insulating coating of the wire and the gap regions. 
When the reinforcement is dry, the gaps are filled with air. However, as the impregnation process progresses, more and more of the gaps are filled with resin. It is expected that the difference in electrical properties between the air that initially fills the gaps and the liquid resin that replaces it will result in a gradual change in electrical response as filling progresses.

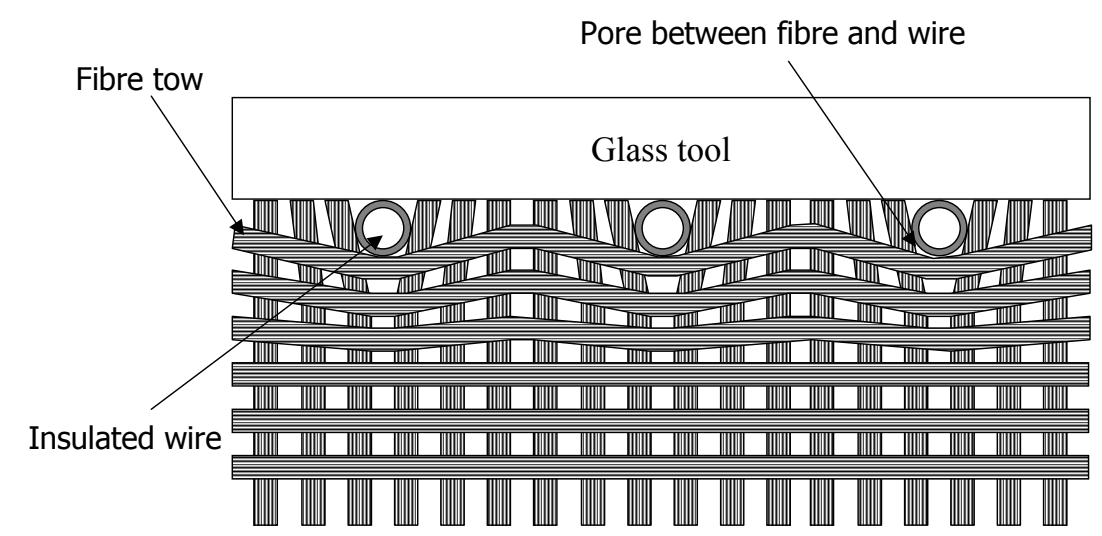

Fig.1 Sensing configuration for resin flow monitoring in carbon fibre reinforcements.

\subsection{Equivalent circuit representation of the sensor response}

In order to gain an insight into the response of the sensor, it is necessary to make an analysis of the various elements of the system, by treating them as components of an electrical circuit. The sensing configuration can be considered as the sum of a number of wire and preform segments with length unity. Assuming that the carbon reinforcement is under uniform potential, all the segments corresponding to one wire are connected in parallel. Each of these segments contains (two types of elements) two elements of different type which are connected in series, one corresponding to the insulating coating and one to the gaps. If the unit length is large compared with the size of the gaps, the impedance of each element corresponding to gaps will be equal to $z_{d}$ for all of the $n$ segments in the dry region of the mould, and equal to $z_{w}$ for all of the $m$ segments in the wetted region. This situation can be represented by the circuit shown in Fig. 2a, which reduces to the circuit represented in Fig. $2 b$.

The circuit in Fig. $2 b$ is equivalent to the circuit presented in [18], which expresses the behaviour of a dielectric sensor designed for glass reinforcements. Consequently, the admittance of the sensor can be expressed as follows [18]:

$$
Y_{\text {sensor }}=m\left(\frac{z_{d}-z_{w}}{z_{c}^{2}+z_{c} z_{d}+z_{c} z_{w}+z_{w} z_{d}}\right)+\left(\frac{(m+n) z_{c}+(m+n) z_{w}}{z_{c}^{2}+z_{c} z_{d}+z_{c} z_{w}+z_{w} z_{d}}\right)
$$


where denotes the impedance per unit length of the insulating layer of the sensing wire. As in the case of a composite containing glass reinforcement, a linear expression can be derived for the admittance of the sensor as a function of the length of wire in contact with liquid resin.

$$
l_{w}=\frac{Y_{\text {sensor }}-Y_{d r y}}{Y_{c o v}-Y_{d r y}} l_{t}
$$
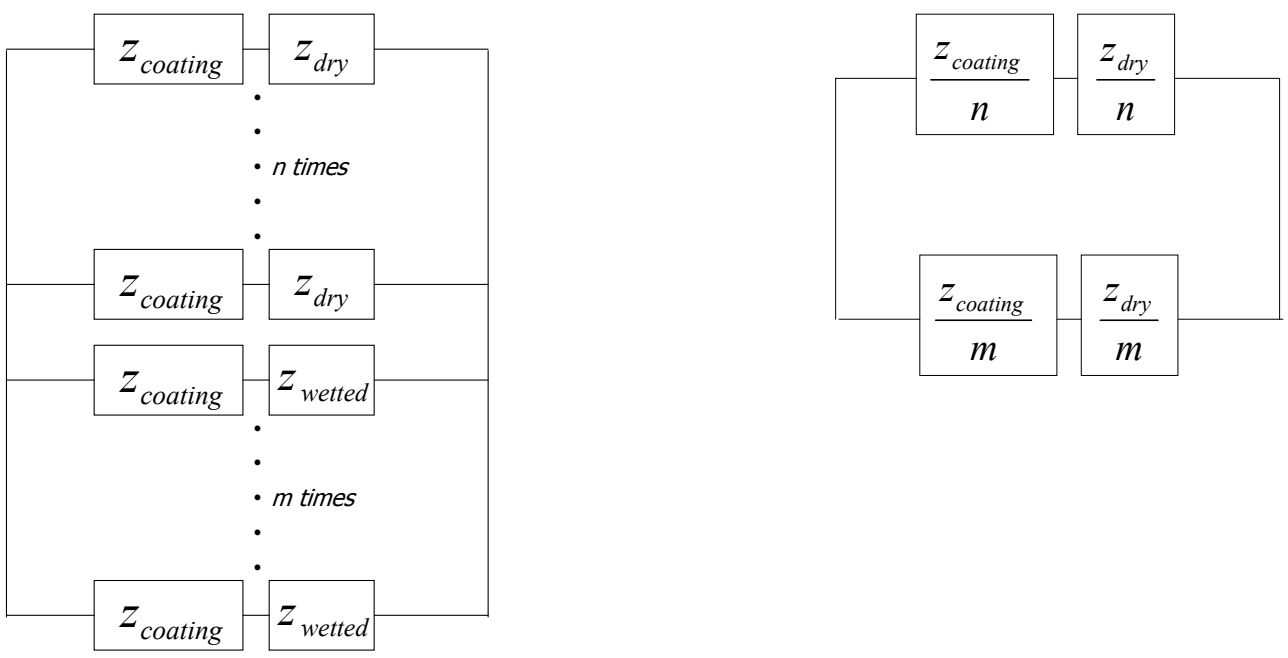

(a)

(b)

Fig.2 Equivalent circuit representation of the sensing setup response.

\section{Flow and cure monitoring performance of the sensor}

\subsection{Experimental details}

The performance of the sensor was validated during the filling and curing of a continuous carbon fibre/RTM6 composite in a partially transparent RTM tool. The non-crimped fabric carbon reinforcement (CTLX, BTI Europe) had a surface density of $816 \mathrm{~g} / \mathrm{m} 2$ and comprised three plies with $+45 /-45 / 0$ orientations. Four layers of this fabric were used, making the total lay-up sequence $(+45 /-45 / 0 / 0 /-45 /+45) 2 \mathrm{~S}$. The fibre weight fraction in the laminate was $69 \%$. The sensor comprised an array of three insulated (polyurethane coated) copper wires, each with a diameter of $0.25 \mathrm{~mm}$, placed at the centre of the mould in contact with the glass top tooling. The distance between the wires was $2 \mathrm{~mm}$. The sensor length was $28 \mathrm{~cm}$ and the mould thickness 3.3 $\mathrm{mm}$. Filling was performed at $120 \mathrm{oC}$. Admittance data were gathered using a Solartron 1260 impedance analyser. The position of the flow front was observed visually as a function of time. After completion of the filling stage, the mould temperature was increased to $160 \mathrm{oC}$ to cure the 
composite. A microdielectrometry experiment using commercial (GIA, Switzerland) comb microelectrodes was performed to monitor the cure of unreinforced RTM6 resin, which was subjected to a thermal programme designed to match that used for cure of the composite in the RTM mould.

\subsection{Flow monitoring results}

Equation 2 was used to calculate the position of the flow front from the admittance data. The admittance measured when the sensor is fully covered by resin is designated as . The calculated length is a complex number. Data on the real and imaginary parts of the length and the visuallyobserved position of the flow front are shown in Fig. 3. Average errors in the location of the flow front and in the imaginary length are given in Table 1. It can be seen that data gathered at 1 $\mathrm{MHz}$ correspond very closely to the visual measurements on the position of the flow front. Data obtained at a lower frequency $(10 \mathrm{kHz})$ show some deviation. The imaginary length has a higher value at the frequency where the error in estimating the location of the flow front (location)** is higher, indicating deviation from linearity as described by Eq. 2. Thus, as in the sensing setup for non-conductive reinforcements [18], the imaginary length offers an independent method for estimating the accuracy of the measurement.

Table 1 Average error in flow front determination and average imaginary length.

\begin{tabular}{l|ll} 
& $10 \mathrm{kHz}$ & $1 \mathrm{MHz}$ \\
\hline Average absolute imaginary length $(\mathrm{cm})$ & 0.38 & 0.20 \\
Average error in flow front location $(\mathrm{cm})$ & 1.98 & 0.84
\end{tabular}




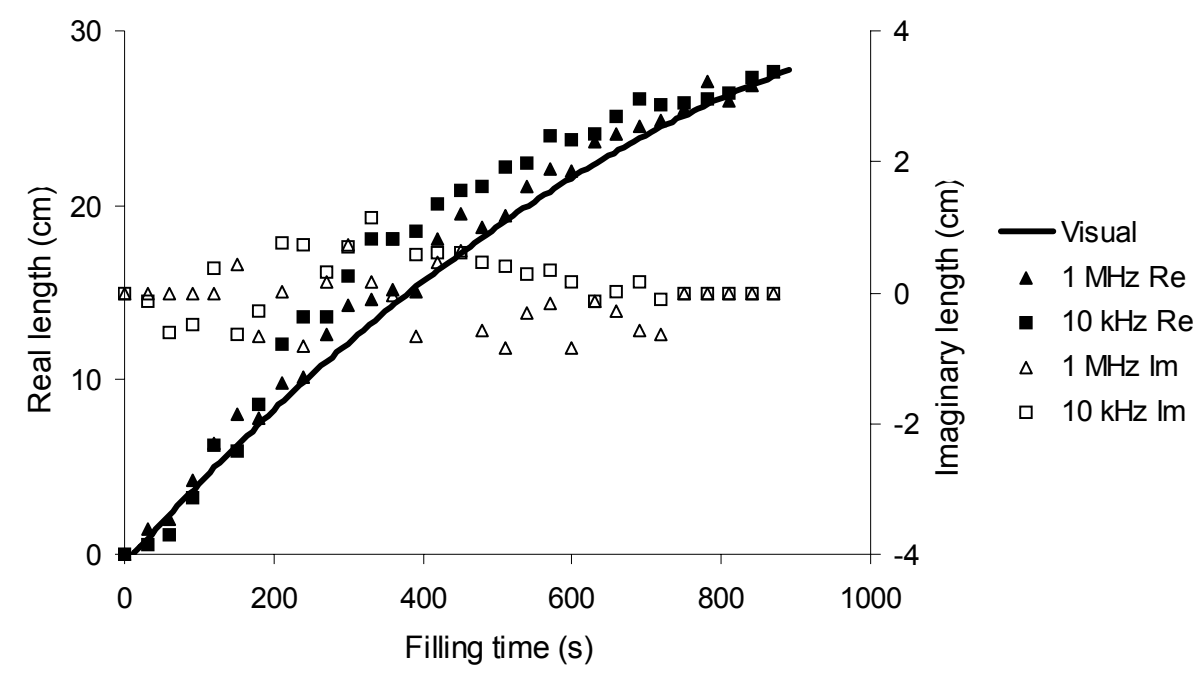

Fig.3 Comparison of visual measurement with dielectric flow measurement for the filling during resin transfer moulding of an carbon/RTM6 fibre composite.

\subsection{Cure monitoring results}

Cure monitoring results obtained using the new sensing configuration are illustrated in Fig.4, which shows the evolution of the normalized imaginary impedance at $10 \mathrm{kHz}$. Normalized impedance is calculated as follows:

$$
\text { Normalized } \log Z^{\prime \prime}=\frac{\log _{10} Z^{\prime \prime}-\log _{10} Z^{\prime \prime}{ }_{\min }}{\log _{10} Z^{\prime \prime}{ }_{\max }-\log _{10} Z^{\prime \prime}{ }_{\min }}
$$

where $Z^{\prime \prime}{ }_{\text {max }}, Z^{\prime \prime}{ }_{\text {min }}$ are the maximum and minimum imaginary impedance values measured during the cure. 


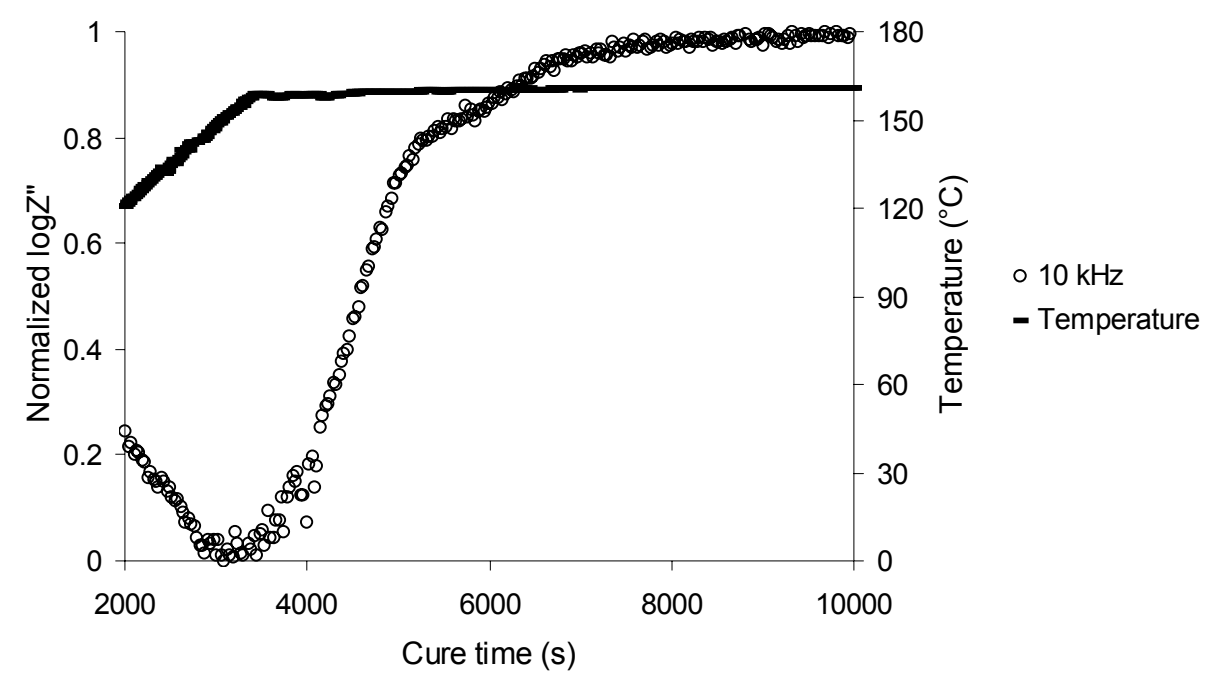

Fig.4 Temperature and normalized impedance at $10 \mathrm{kHz}$ versus cure time using the flow monitoring sensing setup in a carbon/RTM6 fibre composite moulding.

The results of the microdielectrometry experiment are given in Fig.5. By comparing the results, it can be observed that the new sensing arrangement has a response equivalent to that of the microelectrodes. Normalized impedance undergoes a step change during cure, and vitrification (is manifest) manifests itself as a knee in the curve towards the end of the reaction [19]. In comparison with measurements carried out during the cure of the unreinforced RTM6 resin, vitrification is observed at a later cure time in the RTM mould. This is because the flow sensing system performs measurements on the topside of the curing component, where the temperature is lower than the bottom of the moulding. The sensor for measuring and controlling the mould temperature is located at the bottom of the mould. 


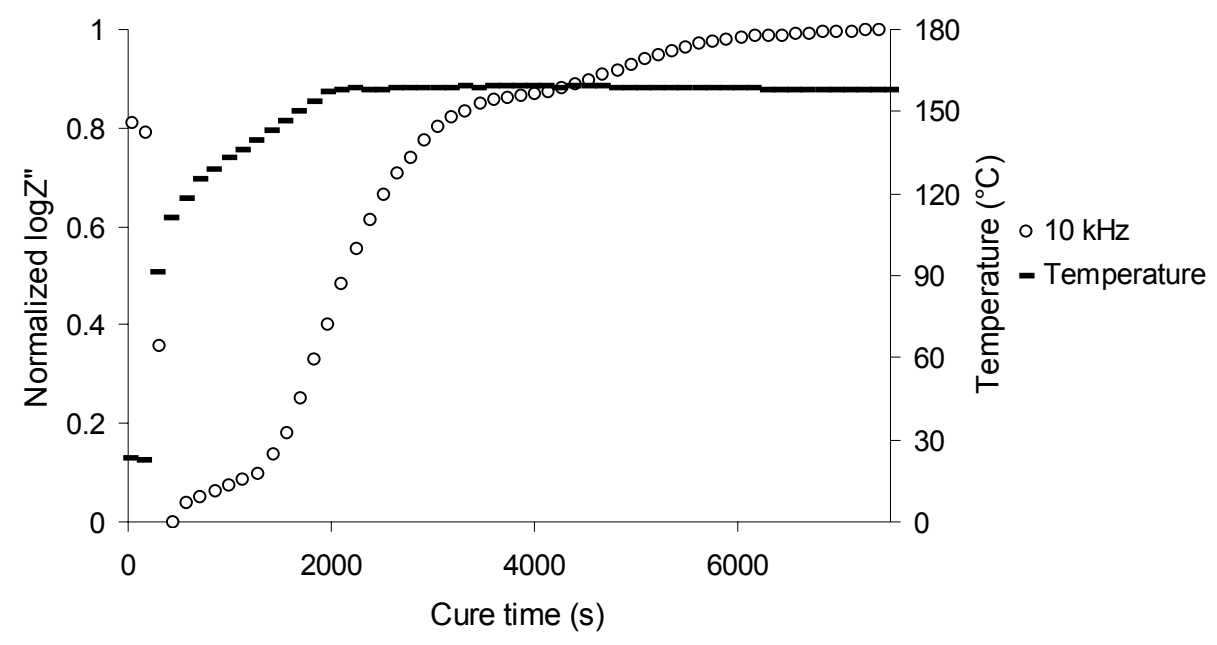

Fig.5 Temperature and normalized impedance at $10 \mathrm{kHz}$ versus cure time obtained using GIA microelectrodes in RTM6 resin.

\section{Conclusions}

A dielectric sensing system for following the resin flow front in liquid moulding of carbon fibre reinforced composites has been developed and tested. Experiments confirm that there is a linear relationship between the admittance of the sensor and the progress of filling, as predicted by a theoretical analysis of the electrical response of the system. The existence of an imaginary part of the measured length gives an estimate of the sensor's deviation from linear behaviour, thus enabling a cross-check to be carried out on the quality of the measurement. The specific monitoring arrangement yields results equivalent to those obtained from conventional microdielectrometry during the curing stage of the process.

\section{Acknowledgements}

The authors wish to acknowledge financial support by the EU (Grant No ERBFMBICT982896). Numerous helpful discussions with Professor (Emeritus) H Block are gratefully acknowledged. 


\section{References}

[1] Liu B C, Bickerton S and Advani S G 1996 Modelling and simulation of resin transfer moulding (RTM)-gate control, venting and dry spot prediction Composites, 27A 135-141

[2] Mal O, Couniot A and Dupret F 1998 Non-isothermal simulation of the resin transfer moulding process Composites 29A 189-198

[3] Rudd C D, Rice E V Bulmer L J and Long A C 1993 Process modelling and design for resin transfer moulding Plastics, Rubber and Composites Processing and Applications 20 67-76

[4] Lekakou C and Bader M G 1998 Mathematical modelling of macro- and micro-infiltration in resin transfer moulding Composites 29A 29-37

[5] Parnas R S, Salem A J, Sadiq T A K, Wang H S and S. G. Advani S G 1994 The interaction between micro- and macro-scopic flow in RTM preforms Composite Structures 27 93-107

[6] Ferland P, Guittard D and Trochu F 1996 Concurrent methods for permeability measurement in resin transfer molding Polymer Composites 17 149-158

[7] Mogavero J and Advani S G 1997 Experimental investigation of flow through multi-layered preforms Polymer Composites 18 649-655

[8] Chan A W, Larive D E and Morgan R J 1993 Anisotropic permeability of fiber preform: Constant flow measurement Journal of Composite Materials 27 996-1008

[9] Gebart B R and Lidstrom P 1996 Measurement of in-plane permeability of anisotropic fiber reinforcements Polymer Composites 17 43-51

[10] Hammami A, Trochu F, Gauvin R and Wirth S 1996 Directional permeability measurement of deformed reinforcement Journal of Reinforced Plastics and Composites 15 552-562

[11]Calchoun D R, Yalvac S, Wetters D G, and Raeck C A 1996 Critical issues in model verification for the resin transfer molding process Polymer Composites 17 11-22

[12]Lundstrom T S, Stenberg R, Bergstrom R, Partanen H and Birkeland P A 2000 In-plane permeability measurements: a nordic round-robin study Composites 31A 29-43

[13] Kranbuehl D E, Kingsley P, Hart S, Hasko G, Dexter B and Loos A C 1994 In situ sensor monitoring and intelligent control of the resin transfer molding process Polymer Composites $15299-305$ 
[14] Schwab S D, Levy R L and Glover G G 1996 Sensor system for monitoring impregnation and cure during resin transfer molding Polymer Composites 17 312-316

[15] Shepard D D 1998 Resin flow front monitoring saves money and improves quality SAMPE Journal 34 31-35

[16]Barooah P, Berker B and Sun J Q 1998 Lineal sensors for liquid injection molding of advanced composite materials Journal of Materials Processing and Manufacturing Science 6 $169-184$

[17] Mathur R, Advani S G, Parnas R S and Fink B K 1997 Evanescent wave fluorescence fiber optic flow sensor for resin transfer molding Proceedings of the $29^{\text {th }}$ SAMPE Technical Conference (Orlando, USA)

[18] Skordos A A, Karkanas P I, and Partridge I K 2000 A dielectric sensor for mesuring flow in resin transfer moulding Measurement Science and Technology 11 25-31

[19] Andjelic S, Mijovic J and Bellucci F 1998 Impedance spectroscopy of reactive polymers. 5. Impedance as a measure of chemical and physical changes in glass formers Journal of Polymer Science: Part B: Polymer Physics 36 641-653 